\title{
miR-30a-3p inhibits the proliferation of liver cancer cells by targeting DNMT3a through the PI3K/AKT signaling pathway
}

\author{
QIONG CHEN ${ }^{1}$, YUAN GAO $^{1}$, QIN YU $^{2}$, FENG TANG $^{3}$, PEI-WEI ZHAO ${ }^{4}$, \\ SU-KUN LUO ${ }^{4}$, JU-SHENG LIN $^{2}$ and HONG MEI ${ }^{1}$ \\ ${ }^{1}$ Department of Gastroenterology, Wuhan Children's Hospital; ${ }^{2}$ Department of Gastroenterology, Tongji Hospital; \\ ${ }^{3}$ Department of Laboratory Medicine; ${ }^{4}$ Clinical Research Center, Wuhan Children's Hospital, Tongji Medical College, \\ Huazhong University of Science and Technology, Wuhan, Hubei 430016, P.R. China
}

Received March 5, 2018; Accepted March 28, 2019

DOI: $10.3892 / \mathrm{ol} .2019 .11179$

\begin{abstract}
MicroRNAs (miRNAs or miRs) are crucial for normal development and maintenance of homeostasis. Dysregulated miRNA expression contributes to numerous pathological conditions, including cancer tumorigenesis. However, a limited number of studies have examined the regulatory effects of miR-30a-3p in tumorigenesis. Therefore, the present study investigated the mechanistic process of tumorigenesis in liver cancer. The results revealed a high expression of DNA methyltransferase 3a (DNMT3a) and a low expression of miR-30a-3p in HepG2 cells compared with that in the L02 cell line. A luciferase reporter assay demonstrated that DNMT3a is a direct target of miR-30a-3p. In addition, DNMT3a overexpression significantly enhanced cell proliferation, which was reversed by a miR-30a-3p mimic. Similarly, the miR-30a-3p mimic blocked DNMT3a-triggered cell cycle processes and apoptosis by attenuating active p-AKT and p-PI3K in HepG2 cells. In summary, the results of the present study demonstrate that miR-30a-3p is essential for cell proliferation regulation via its association with $\mathrm{AKT} / \mathrm{PI} 3 \mathrm{~K}$ signaling in liver cancer. These results provide insight into the molecular mechanism by which miR-30a-3p inhibits liver cancer cell proliferation and provides a foundation for its clinical development and application.
\end{abstract}

\section{Introduction}

Liver cancer is one of the most common tumor types, is the third leading cause of cancer-associated mortality worldwide, and consists of hepatocellular carcinoma (HCC) and

Correspondence to: Ms. Qiong Chen, Department of Gastroenterology, Wuhan Children's Hospital, Tongji Medical College, Huazhong University of Science and Technology, 100 Hongkong Road, Wuhan, Hubei 430016, P.R. China

E-mail: 891206515@qq.com

Key words: miRNA-30a-3p, DNA methyltransferase 3a, liver cancer, cell proliferation, PI3K/AKT signaling pathway hepatoblastoma (HB) (1). HCC and HB have distinct cytological characteristics (2). HepG2 was originally thought to be a HCC cell line; however, was misidentified and has subsequently been demonstrated to be derived from hepatoblastoma (3). While liver resection, radiofrequency ablation and transplantation are efficient treatments for patients with liver cancer, the prognosis of the majority of patients remains poor (4). Therefore, an investigation of the underlying mechanism of liver cancer is urgently required in order to identify novel therapeutic targets.

DNA methyltransferases (DNMTs) are responsible for establishing de novo genomic DNA methylation patterns involved in covalent addition of a methyl group to the carbon atom 5 of the cytosine pyrimidine ring in a $\mathrm{CpG}$ (cytosine-guanine) dinucleotide (5). Methyl groups in dinucleotide $\mathrm{CpGs}$ of gene promoters regulate interactions between DNA and the transcription machinery of the cell (6). Cui et al (7) demonstrated that DNMT and microRNAs (miRNAs/miRs) participate in gastric cancer migration and invasion. Several studies have also demonstrated that DNMT and microRNAs regulate tumorigenesis (8-10).

MicroRNAs (miRNAs) are a large set of small non-coding RNAs, approximately 22 nucleotides in length, which primarily bind to seed sequences located within the 3 ' untranslated region (3'-UTR) of target mRNAs (11). Their structures are highly conserved and regulate gene expression post-transcriptionally by inhibiting protein translation or promoting the degradation of target mRNAs $(12,13)$. miRNAs abrogate the biological functions of their target genes (14). Over 2,500 human miRNAs have been identified to serve critical roles in various physiological and pathological processes, including embryonic development, angiogenesis, apoptosis, autophagy, cell cycle progression and cell proliferation (15-17).

Due to their abundance, an increasing number of studies have suggested that miRNAs exert pivotal functions in human tumorigenesis and development, particularly in liver cancer (18-20). It has been reported that certain miRNAs, including let-7g, miR-24a, miRNA-30a-3p, miRNA-138 miR-203 and miR-451, are associated with tumor progression $(21,22)$. Of note, the PI3K/AKT signaling pathway is considered a canonical regulator of tumorigenesis (23). Furthermore, negative feedback regulation occurs between 
miRNAs and DNMTs (24). Therefore, the present study examined whether these miRNAs and DNMTs are associated with the PI3K/AKT signaling pathway in liver cancer cells.

To the best of our knowledge, no previous studies have examined the role of miRNA-30a-3p in liver cancer. Therefore, the present study investigated the detailed mechanism of how miRNA-30a-3p and DNMT3A contribute to cell proliferation in liver cancer cells.

\section{Materials and methods}

Cell culture. The hepatoblastoma cell line HepG2 and a normal liver cell line L02 were obtained from Shanghai Institute for Biological Sciences, Chinese Academy of Science (Shanghai, China). Cells were cultured at $37^{\circ} \mathrm{C}$ in $5 \% \mathrm{CO}_{2}$ in Dulbecco's modified Eagle's medium containing $10 \%(\mathrm{v} / \mathrm{v})$ fetal bovine serum (Gibco; Thermo Fisher Scientific, Inc., Waltham, MA, USA), $100 \mu \mathrm{g} / \mathrm{ml}$ penicillin, $100 \mu \mathrm{g} / \mathrm{ml}$ streptomycin (Gibco; Thermo Fisher Scientific, Inc.).

Cell transfection. The miR-30a-3p mimic (miR-30a-3p; sequence, 5'-CUUUCAGUCGGAUGUUUGCAGC-3') and negative control (miR-NC; sequence, 5'-UUUGUACUACACAAA AGUACUG-3') were designed and synthesized by Guangzhou RiboBio, Co., Ltd. (Guangzhou, China). HepG2 cells were transfected with $100 \mathrm{nM}$ of the chemically synthesized miR-30a-3p mimic and miR-NC using Lipofectamine ${ }^{\circledR} 2000$ (Invitrogen; Thermo Fisher Scientific, Inc.), according to the manufacturer's protocol. Furthermore, the DNMT3a stable overexpression vector pCMV6-AC-DNMT3a (termed DNMT3a; $0.8 \mu \mathrm{g}$; Myhalic Biotechnology Co., Ltd., Wuhan, China,) and vector control (termed Vector; $0.8 \mu \mathrm{g}$; Myhalic Biotechnology Co., Ltd.) were transfected into HepG2 cells using Nucleofector ${ }^{\circledR}$ Program X-01 (Lonza Group, Ltd., Basel, Switzerland), according to the manufacturer's protocol. Additionally, a co-transfection group was transfected with DNMT3a and miR-30a-3p (termed DNMT3a+miR-30a-3p). The co-transfection group was transfected with vector using the electroporation method (25) after cells were stably transfected miRNA mimics with Lipofectamine 2000. miRNA mimics were not transfected with the electroporation method as Lipofectamine 2000 has a significantly increased transfection efficiency (26). After the cells were transfected with different treatments for $48 \mathrm{~h}$ they were cultured for subsequent experiments.

Reverse transcription-quantitative polymerase chain reaction $(R T-q P C R)$. Total RNA was extracted from LO2 cells and HepG2 cells transfected with miR-30a-3p mimic, DNMT3a or controls using TRIzol ${ }^{\circledR}$ reagent (Invitrogen; Thermo Fisher Scientific, Inc.), according to the manufacturer's protocol. RT for miR-30a-3p was performed using Hairpinit ${ }^{\mathrm{TM}} \mathrm{miR}$ qPCR Quantification kit (Shanghai GenePharma, Co., Ltd., Shanghai, China). RT for DNMT3a was performed using the PrimeScript ${ }^{\mathrm{TM}}$ RT Reagent kit (Takara Biotechnology Co., Ltd., Dalian, China). qPCR was performed using SYBR green (Takara Biotechnology Co., Ltd.) as a fluorophore. The following primers were used for qPCR: miR-30a-3p forward, 5'-CTTTCAGTCGGATGTTTGCAGC-3', and reverse, 5'-ACA CTCCAGCTGGGCTTTCAGTCGGATG-3'; DNMT3a forward, 5'-AGGCTGGAATGTAGTGGTACATCA-3', and reverse, 5'-AGGGTGGGAGGATCGCTTGA-3'; U6 forward, 5'-CTCGCTTCGGCAGCACA-3' and reverse, 5'-AACGCT TCACGAATTTGCGT-3'; and GAPDH forward, 5'-CAA GTTCAACGGCACAG-3' and reverse, 5'-CCAGTAGACTCC ACGACAT-3'. U6 and GAPDH were used as controls. The thermocycling conditions were as follows: $95^{\circ} \mathrm{C}$ for $5 \mathrm{~min}$, $95^{\circ} \mathrm{C}$ for $15 \mathrm{sec}$ and $60^{\circ} \mathrm{C}$ for $1 \mathrm{~min}$, with a total of 39 cycles of amplification. The results were quantified using the $2^{-\Delta \Delta C q}$ analysis method (27).

Luciferase reporter assay. TargetScan (www.targetscan.org) was used to identify potential targets of miR-30a-3p. An important enzyme in DNA methylation (28), DNMT3a, was identified as a potential target of miR-30a-3p. The putative target sites of the human DNMT3a 3'-UTR segments for miR-30a-3p were amplified by PCR (29). The sequences of wild-type DNMT3a 3'-UTR and mutant DNMT3a 3'-UTR were 5'-AAAGGG UUGGACAUCAUCUCC-3' and 5'-AAAGGGUUGGACAAG UAGAGC-3', respectively. The PCR product was inserted into the luciferase reporter pGL3 dual luciferase reporter vector (Thermo Fisher Scientific, Inc.) to generate the DNMT3a 3'-UTR reporter (termed pGL3-DNMT3a). Mutant DNMT3a segments were prepared by mutating the seed regions of the miR-30a-3p binding sites using a site-directed mutagenesis kit (Takara Bio, Inc., Otsu, Japan) and termed pGL3-mutant DNMT3a. For reporter assays, $1 \times 10^{6}$ cells were seeded in 24-well plates and co-transfected with $0.25 \mu \mathrm{g}$ NMT3a 3'-UTR or mutant DNMT3a 3'-UTR and $50 \mathrm{nM}$ miR-30a-3p mimic or NC using Lipofectamine ${ }^{\circledR} 2000$ (Thermo Fisher Scientific, Inc.). Each transfection was performed in triplicate and luciferase activity was measured using the Dual-Luciferase Reporter assay system (Promega Corporation, Madison, WI, USA) following transfection for $48 \mathrm{~h}$. The results were normalized to Renilla luciferase activity.

Assessment of apoptosis and cell cycle distribution by flow cytometry. Cell apoptosis was determined using the Annexin V-FITC/propidium iodide (PI) apoptosis detection kit (Bioswamp; Myhalic Biotechnology Co., Ltd.), according to the manufacturer's protocol. Briefly, HepG2 cells $\left(1 \times 10^{6}\right)$ were collected, washed twice with PBS and then resuspended in $100 \mu \mathrm{l} 1 \mathrm{x}$ binding buffer, to which $4 \mu \mathrm{l} \mathrm{PI}$ and $4 \mu \mathrm{l}$ Annexin V-FITC were added, and incubated at room temperature for $30 \mathrm{~min}$ in the dark, after which $500 \mu \mathrm{l} 1 \mathrm{x}$ binding buffer was added and mixed. Apoptotic cells were analyzed by a flow cytometer (FACSCalibur, Becton Dickinson and Company, Franklin Lakes, NJ, USA). The analysis of data was performed using FCS Express V3 (De Novo Software; Glendale, CA, USA).

For cell cycle analysis, HepG 2 cells $\left(1 \times 10^{6}\right)$ were harvested and fixed with $75 \%$ ethanol at $-20^{\circ} \mathrm{C}$ overnight, then washed twice with PBS for $1 \mathrm{~min}$ at room temperature. Following this treatment, $200 \mu \mathrm{l}$ PI was added to the mixture and incubated for $30 \mathrm{~min}$ in the dark at room temperature, followed by filtration with a 200 mesh filter membrane. Cell cycle was determined based on analysis with a flow cytometer. The analysis of data was performed using FCS Express V3 software. Experiments were performed in duplicate.

Cell proliferation analysis. Cell proliferation was measured using a CCK-8 kit (Dojindo Molecular Technologies, Inc., 
Kumamoto, Japan), according to the manufacturer protocol. Briefly, HepG2 cells (4x10 ${ }^{3}$ cells/well) were seeded in 96-well plates (Corning Inc., Corning, NY, USA) and incubated at $37^{\circ} \mathrm{C}$ in a $5 \% \mathrm{CO}_{2}$ atmosphere for $24 \mathrm{~h}$. Subsequently, $10 \mu \mathrm{l}$ CCK-8 solution was added to each well and cultured at $37^{\circ} \mathrm{C}$ for 2- $4 \mathrm{~h}$. The absorbance was then measured at $450 \mathrm{~nm}$ under a microplate reader (Molecular Devices, LLC, Sunnyvale, CA, USA). All experiments were performed in triplicate.

Colony formation assay. HepG2 cells were plated at 400 or 800 cells per well in 6-well plates and incubated for 2 weeks at $37^{\circ} \mathrm{C}$ in a $5 \% \mathrm{CO}_{2}$ humidified environment. Colonies were stained with crystal violet $(0.5 \% \mathrm{w} / \mathrm{v})$ for $30 \mathrm{~min}$ following fixing with pure methanol at room temperature and then photographed using a mobile phone camera, followed by counting. Experiments were performed in duplicate.

Western blot analysis. LO2 cells and $\mathrm{HepG} 2$ cells transfected with miR-30a-3p mimic, DNMT3a or controls were lysed with radioimmunoprecipitation assay (RIPA) lysis buffer (Beyotime Institute of Biotechnology, Haimen, China) containing phenylmethylsulfonyl fluoride and were quantified using the modified Bradford method. Equal amounts of protein samples $(20 \mu \mathrm{g})$ were subjected to $12 \%$ SDS-PAGE and transferred to polyvinylidene difluoride membranes (EMD Millipore, Billerica, MA, USA). The membrane was then incubated with TBS and $0.05 \%$ Tween-20 buffer with 5\% bovine serum albumin (Gibco; Thermo Fisher Scientific, Inc.) for $1 \mathrm{~h}$ at room temperature with gentle shaking. The membranes were subsequently incubated with specific antibodies against DNMT3a (cat. no. AB188470), PI3K (cat. no. AB180967), AKT (cat. no. AB8805), phosphorylated (p)-PI3K (cat. no. AB182651), p-AKT (cat. no. AB38449), cyclin D1 (cat. no. AB134175), cyclin-dependent kinase (CDK2; cat. no. AB32147), p21 (cat. no. AB109199), p27 (cat. no. AB75908) (all 1:1,000; Abcam, Cambridge, UK) or GAPDH (cat. no. AC036, 1:1,000; ABclonal Technology, Wuhan, China) for $2 \mathrm{~h}$ at room temperature. The membrane was subsequently washed and incubated with horseradish peroxidase (HRP)-conjugated secondary antibody goat anti-rabbit IgG (1:1,000; cat. no. PAB150011; Bioswamp; Myhalic Biotechnology Co., Ltd.) for $1 \mathrm{~h}$ at room temperature. Finally, protein bands were detected by a chemiluminescent HRP substrate (EMD Millipore), quantitated by densitometric analysis using Image Lab 3.0 software (Bio-Rad, Laboratories, Inc., Hercules, CA, USA) and expressed as a percentage of the control following normalization to GAPDH.

Statistical analysis. All statistical analyses were performed with GraphPad Prism 6.0 (GraphPad, Inc., La Jolla, CA, USA) or SPSS 19.0 (IBM Corp., Armonk, NY, USA). Results are presented as the mean \pm standard deviation. Comparisons between two groups were analyzed by Student's unpaired t-test. Comparisons between more than two groups were analyzed by one-way ANOVA followed by Bonferroni's post hoc test. $\mathrm{P}<0.05$ was considered to indicate a statistically significant difference.

\section{Results}

DNMT3a and miR-30a-3p are aberrantly expressed in liver cancer cells. To investigate the association between DNMT3a and miR-30a-3p, the cell lines HepG2 and L02 were used in the
A

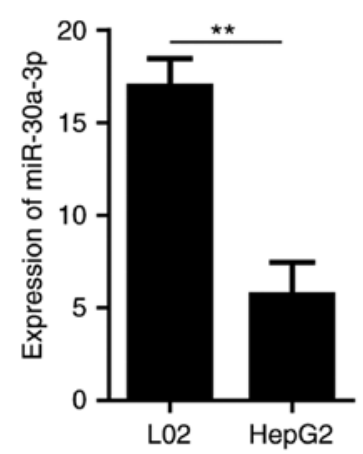

B
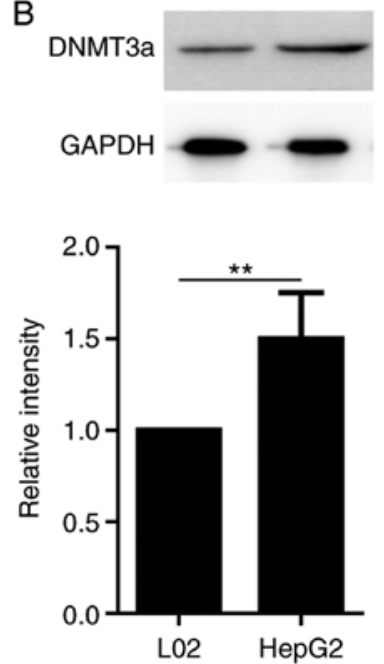

Figure 1. Expression levels of DNMT3a and miR-30a-3p in different hepatocyte cell lines.(A) The expression of miR-30a-3p in L02 and HepG2 cells was measured by RT-qPCR. (B) The levels of DNMT3a were analyzed by western blotting in the L02 and HepG2 cell lines. The data are presented as the mean \pm standard deviation of three independent experiments. ${ }^{* *} \mathrm{P}<0.01$ vs. HepG2. RT-qPCR, reverse transcription-quantitative polymerase chain reaction; DNMT3a, DNA methyltransferase 3a; miR-30a-3p, microRNA-30a-3p.

present study. Total RNA was extracted from these cells and reversed transcribed into cDNA and RT-qPCR was performed to investigate the expression levels of miR-30a-3p. In addition, total protein was extracted to detect the expression of DNMT3a by western blotting. As presented in Fig. 1A, miR-30a-3p expression was significantly lower in the HepG2 cell line compared with L02 cells. Furthermore, DNMT3a protein level was significantly higher in the HepG2 cell line compared with the L02 cell line (Fig. 1B). These results suggest that HepG2 cells express high levels of DNMT3a and low levels of miR-30a-3p compared with the other hepatocyte cells examined. Therefore, HepG2 cells were used in the subsequent assays.

DNMT3a is a direct target of miR-30a-3p in HepG2 cells. To identify potential target genes of miR-30a-3p, the present study used TargetScan to predict the mRNA targets of miR-30a-3p, which revealed that DNMT3a contained a miR-30a-3p recognition site in its 3'-UTR (Fig. 2A). Subsequently, HepG2 cells were transfected with miR-30a-3p mimic and the transfection efficiency was verified by RT-qPCR (Fig. 2B). To establish whether DNMT3a is a direct target of miR-30a-3p, DNMT3a 3'-UTR sequences containing the predicted target site of miR-30a-3p were cloned into a luciferase reporter vector. The results demonstrated that luciferase activity in the group co-transfected with miR-30a-3p and pGL3-DNMT3a reporter was significantly decreased, whereas there was no change in the group transfected with the mutant DNMT3a 3'UTR (Fig. 2C). This result suggests that DNMT3a is direct target of miR-30a-3p.

To further confirm the association between miR-30a-3p and DNMT3a, RT-qPCR and western blot analyses were performed to evaluate the mRNA and protein expression levels of the miR-30a-3p potential target gene DNMT3a. The results revealed that the protein expression of DNMT3a was significantly decreased when miR-30a-3p was overexpressed as compared with cells transfected with miR-NC (Fig. 2D). These results also 
A

\begin{tabular}{|c|c|c|c|}
\hline & $\begin{array}{c}\text { Predicted consequential pairing of target region (top) } \\
\text { and miRNA (bottom) }\end{array}$ & $\begin{array}{l}\text { Site } \\
\text { type }\end{array}$ & $\begin{array}{l}\text { Context++ } \\
\text { score }\end{array}$ \\
\hline $\begin{array}{l}\text { Position } 350-356 \text { of DNMT3A } 3 \text { 'UTR } \\
\text { hsa-miR-30a-3p }\end{array}$ & $\begin{array}{c}5^{\prime} \text {...UUGUUUCUUGCUGUGACUGAAAC... } \\
3^{\prime} \text { CGACGUUUGUAGGCUGACUUUC }\end{array}$ & $\begin{array}{c}7 \mathrm{mer}- \\
\mathrm{m} 8\end{array}$ & -0.02 \\
\hline
\end{tabular}

B

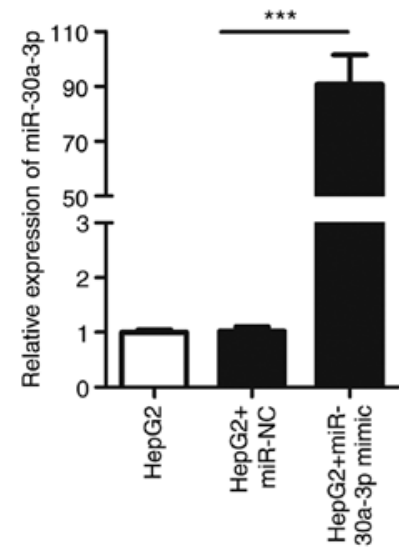

E

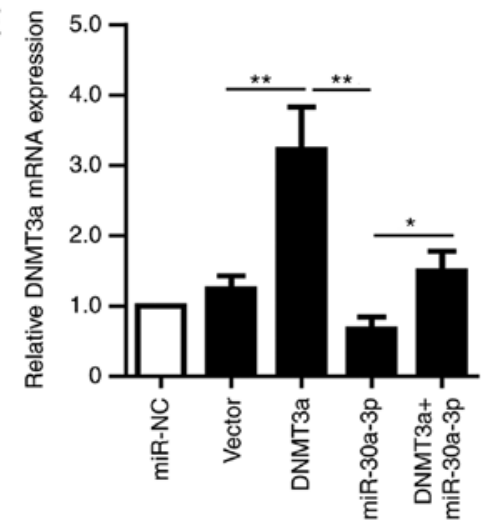

C
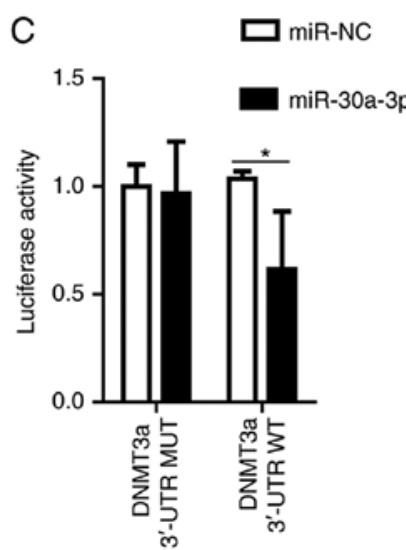

D
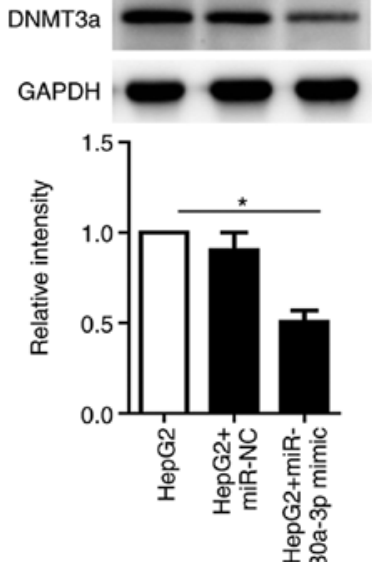

$\mathrm{F}$
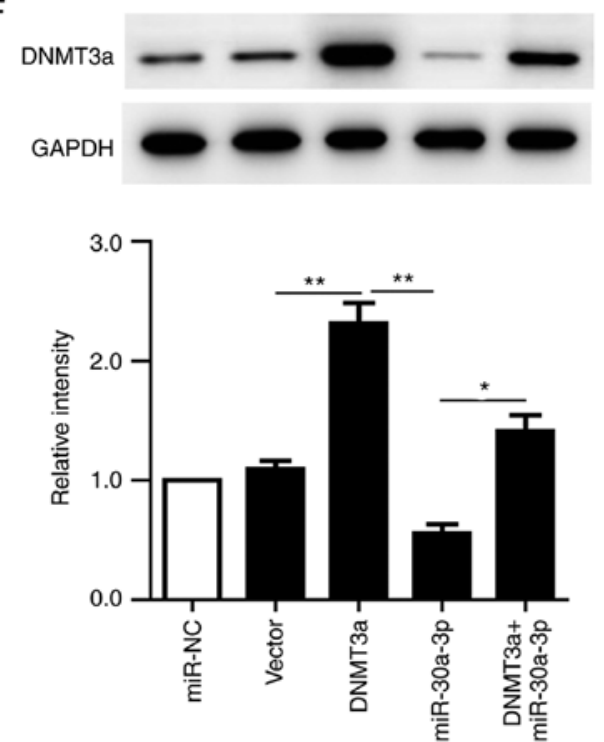

Figure 2. DNMT3a is a direct target of miR-30a-3p in HepG2 cells. (A) DNMT3a was predicted as a potential target of miR-30a-3p by the online program TargetScan. (B) The expression level of miR-30a-3p in transfected HepG2 cells was detected by RT-qPCR. (C) The relative luciferase activity in HepG2 cells detected after the WT or MUT DNMT3A 3'-UTR genes were co-transfected with miR-NC or miR-30a-3p mimic. (D) The expression of DNMT3a was analyzed by western blotting following transfection with miR-30a-3p mimic, miR-30a-3p NC or no transfection. (E) The mRNA levels of DNMT3a were measured by RT-qPCR following transfection with miR-30a-3p mimic, pCMV6-AC-DNMT3a, vector control, co-transfection or no transfection of HepG2 cells. (F) The expression of DNMT3a was analyzed by western blotting following transfection with miR-30a-3p mimic, pCMV6-AC-DNMT3a, vector control, co-transfection or no transfection of HepG2 cells. The data are presented as the mean \pm standard deviation of three independent experiments. ${ }^{*} \mathrm{P}<0.05$. ${ }^{* *} \mathrm{P}<0.01$; ${ }^{* * * *} \mathrm{P}<0.001$. RT-qPCR, reverse transcription-quantitative polymerase chain reaction; NC, negative control; MUT, mutant; WT, wild-type; miR, microRNA; DNMT3a, DNA methyltransferase 3a.

demonstrated that vector and miRNA transfection efficiencies were high, and the DNMT3a mRNA levels were detected by RT-qPCR in different cells following transfection. The results revealed that the co-transfection group demonstrated significantly increased levels of DNMT3a compared with that following transfection with miR-30a-3p alone. Transfection with the miR-30a-3p mimic significantly suppressed the expression level of DNMT3a compared with the group transfected with DNMT3a vector. The protein expression of DNMT3a was consistent with the trend of mRNA levels (Fig. 2E and F). In summary, these results suggest that DNMT3a is a direct target of miR-30a-3p, which suppresses DNMT3a expression in HepG2 cells.
DNMT3a is involved in miR-30a-3p-mediated suppression of cell proliferation. Transfection with pCMV6-AC-DNMT3a markedly increased colony formation compared with the vector group. In addition, transfection with the miR-30a-3p mimic resulted in significantly decreased colony formation, which was rescued by co-transfection (Fig. 3A and B). To further investigate the role of DNMT3a and miR-30a-3p in HepG2, the present study analyzed the proliferation of pCMV6-AC-DNMT3a and miR-30a-3p mimic-treated HepG2 cells in a CCK-8 assay. The results demonstrated that the cell proliferation ability was significantly promoted when cells were transfected with pCMV6-AC-DNMT3a compared with the 
A
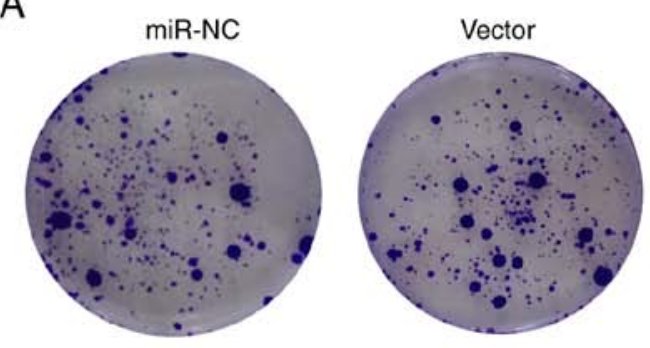

B

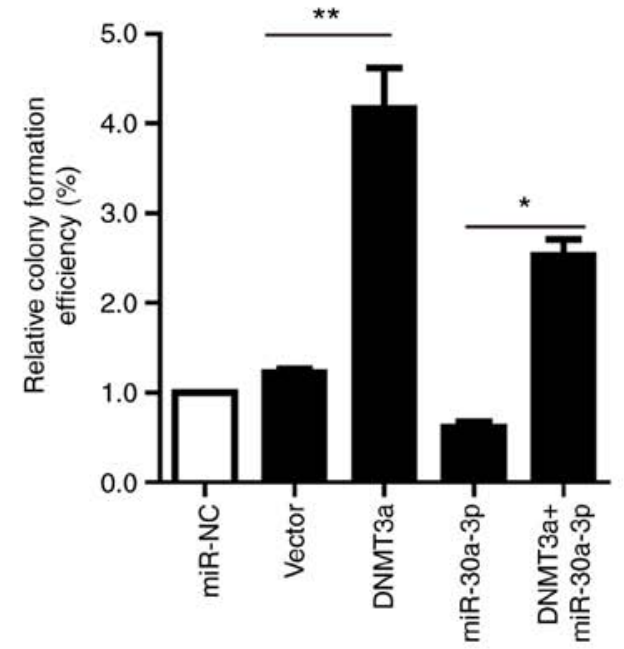

DNMT3a

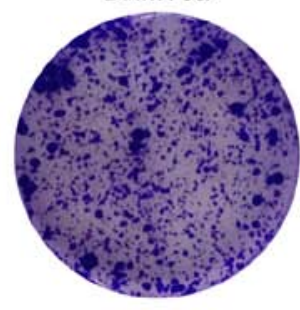

C

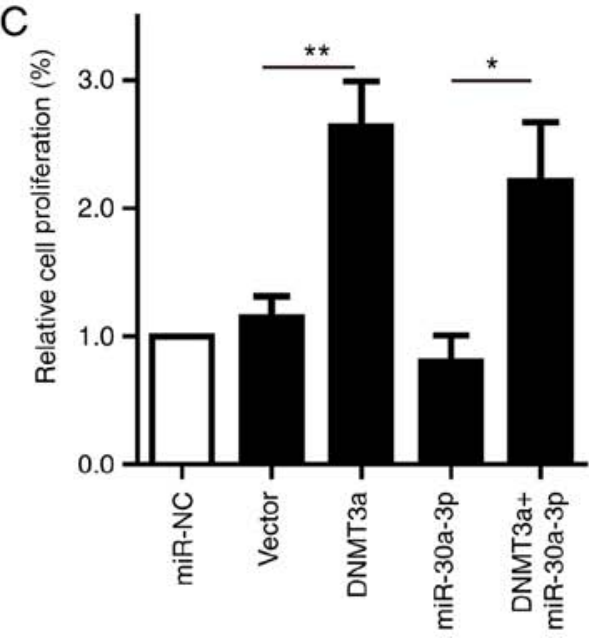

Figure 3. miR-30a-3p and DNMT3a regulate colony formation and proliferation of HepG2 cells. (A) A colony formation assay was performed to examine the colony formation following transfection with the miR-30a-3p mimic, pCMV6-AC-DNMT3a, co-transfection, miR-NC or empty vector (B) Quantification of colony numbers after different treatments. (C) A CCK-8 assay was performed to evaluate the proliferation of HepG2 cells following transfection with miR-30a-3p mimic, pCMV6-AC-DNMT3a, co-transfection, miR-NC or empty vector. The data are presented as the mean \pm standard deviation of three independent experiments. ${ }^{*} \mathrm{P}<0.05,{ }^{* *} \mathrm{P}<0.01$. NC, negative control; miR, microRNA; DNMT3a, DNA methyltransferase 3a.

vector control group. However, cell proliferation was markedly suppressed when the miR-30a-3p mimic was transfected into HepG2 cells compared with either the miR-NC or DNMT3a group. Furthermore, HepG2 cells in the co-transfection group exhibited significantly enhanced proliferation compared with the group transfected with miR-30a-3p mimic alone (Fig. 3C). Thus, the results of the present study further support that DNMT3a is a direct target of miR-30a-3p and that miR-30a-3p inhibits the colony formation and proliferation of HepG2 cells.

Overexpression of $\mathrm{miR}-30 \mathrm{a}-3 \mathrm{p}$ reverses the effect of DNMT3a and inhibits HepG2 cell cycle progression. To determine the role of miR-30a-3p in the cell cycle, HepG2 cells were transfected with the miR-30a-3p mimic, pCMV6-AC-DNMT3a, miR-NC, an empty vector or co-transfected with miR-30a-3p mimic and pCMV6-AC-DNMT3a. Cell cycle progression was examined by flow cytometry. The results demonstrated that the proportion of G1 phase cells was markedly increased, while the proportion of $\mathrm{S}$ phase cells was decreased when cells were transfected with miR-30a-3p mimic compared with miR-NC. However, cell transfection with pCMV6-AC-DNMT3a demonstrated that the proportion of G1 phase cells was markedly decreased, while the proportion of $\mathrm{S}$ phase cells was increased compared with that in the vector group (Fig. 4A and B). Furthermore, to further evaluate the potential role of miR-30a-3p in the cell cycle of HepG2 cells, expression of the cell cycle-associated proteins p21, p27, cyclin D1 and CDK2 were analyzed by western blotting following transfection with miR-30a-3p mimic, pCMV6-AC-DNMT3a or co-transfection. The results revealed that cyclin D1 and CDKs were significantly increased, while the levels of p21 and p27 were significantly decreased following transfection with pCMV6-AC-DNMT3a compared with the vector control group. However, transfection of miR-30a-3p mimic into HepG2 cells upregulated the expression of p21 and p27 and decreased the expression of cyclin D1 and CDK2 compared with the miR-NC group. Simultaneously, co-transfection significantly increased the protein levels of cyclin D1 and CDKs and significantly decreased p21 and p27 compared with the cells transfected with the miR-30a-3p mimic alone (Fig. 4C-G). These results indicate that miR-30a-3p is involved in cell cycle regulation, which may occur with the help of the cycle-associated proteins that block the G0/G1 phase.

Overexpression of miR $-30 a-3 p$ facilitates apoptosis via suppression of the PI3K/AKT signaling pathway. The present study examined whether the miR-30a-3p mimic affects the level of apoptotic HepG2 cells following different treatments. The results revealed the proportions of Annexin V-FITC-positive/propidium iodide-positive and -negative cells, indicating the presence of late apoptotic and early apoptotic cells. Treatment with pCMV6-AC-DNMT3a was observed to significantly reduced cell apoptosis compared with the vector control. Conversely, treatment with the miR-30a-3p mimic significantly induced apoptosis compared with the miR-NC and DNMT3a groups. Co-transfection slightly reversed the 

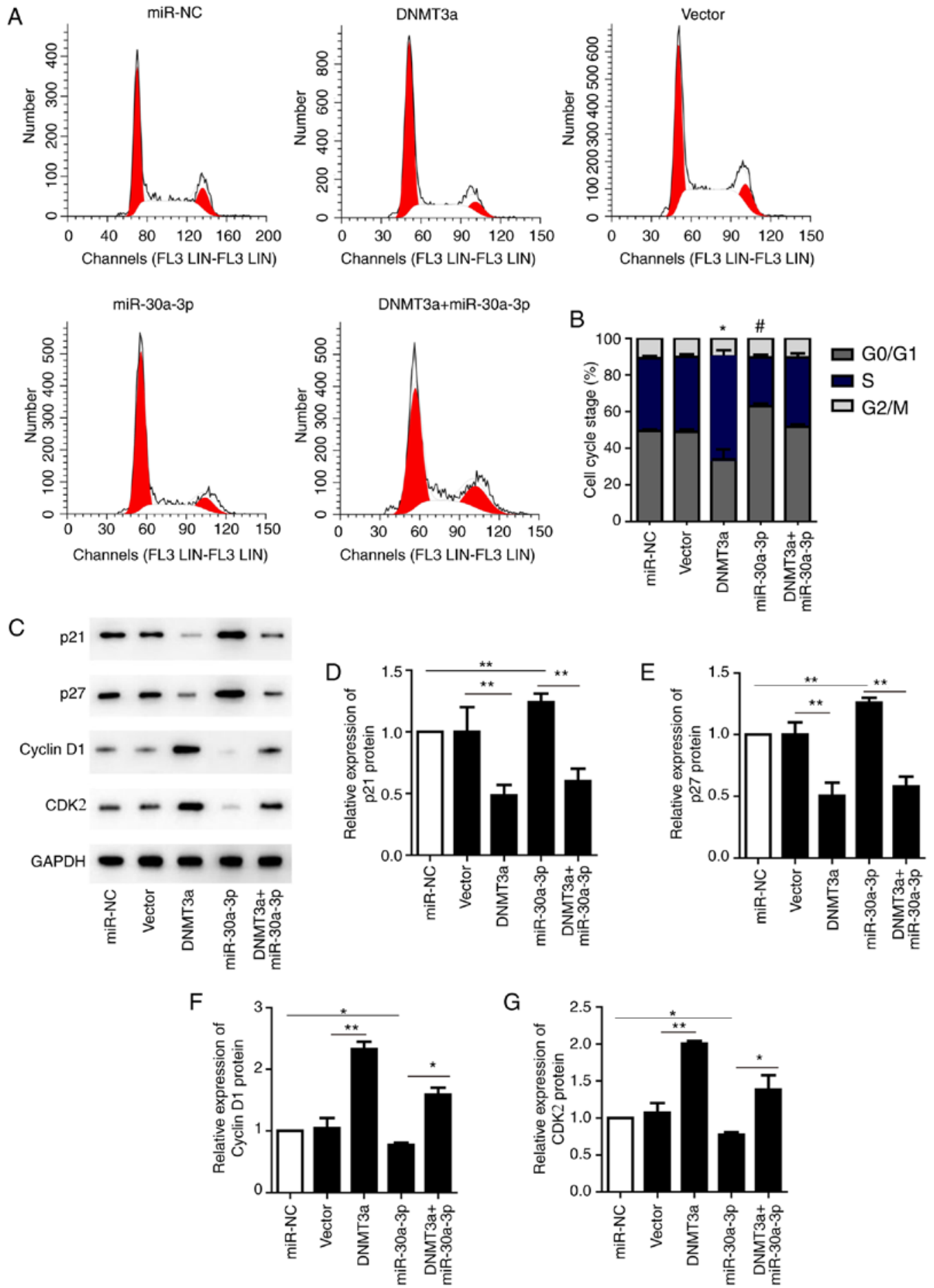

Figure 4. Overexpression of miR-30a-3p reverses the DNMT3a-induced effects on the cell cycle process of HepG2 cells. (A) Following transfection with the miR-30a-3p mimic, pCMV6-AC-DNMT3a, co-transfection, miR-NC and empty vector, the cells were analyzed by flow cytometry. (B) Bar graph represents the percentage distribution of HepG2 cells in different phases of the cell cycle. "P $<0.05 \mathrm{G} 1$ and $\mathrm{S}$ phase of DNMT3a vs. G1 and $\mathrm{S}$ phase Vector. ${ }^{\prime \prime} \mathrm{P}<0.05 \mathrm{G} 1$ and S phase of miR-30a-3p vs. G1 and S phase of miR-NC. (C) The cell cycle associated proteins p21, p27, cyclin D1 and CDK2 were analyzed by western blotting. Relative expression of (D) p21, (E) p27, (F) cyclin D1 and (G) CDK2 were quantified and analyzed statistically. ${ }^{*} \mathrm{P}<0.05$, ${ }^{* * *} \mathrm{P}<0.01$. The data are presented as the mean \pm standard deviation of three independent experiments. NC, negative control; miR, microRNA; DNMT3a, DNA methyltransferase 3a; CDK2, cyclin-dependent kinase 2 .

apoptotic rate compared with transfection with miR-30a-3p mimic alone (Fig. 5A and B). Thus, the trend was similar to the alterations observed in the cell cycle.

In addition, the levels of p-AKT and p-PI3K were significantly increased in the cells transfected with pCMV6-AC-DNMT3a compared with the vector group.
Simultaneously, the expression levels of p-AKT and p-PI3K were significantly decreased in the cells transfected with the miR-30a-3p mimic compared with the miR-NC group; this effect could be significantly reversed by co-transfection, and the increase in the expression levels of p-AKT and p-PI3K was clearly associated with the transfection of 

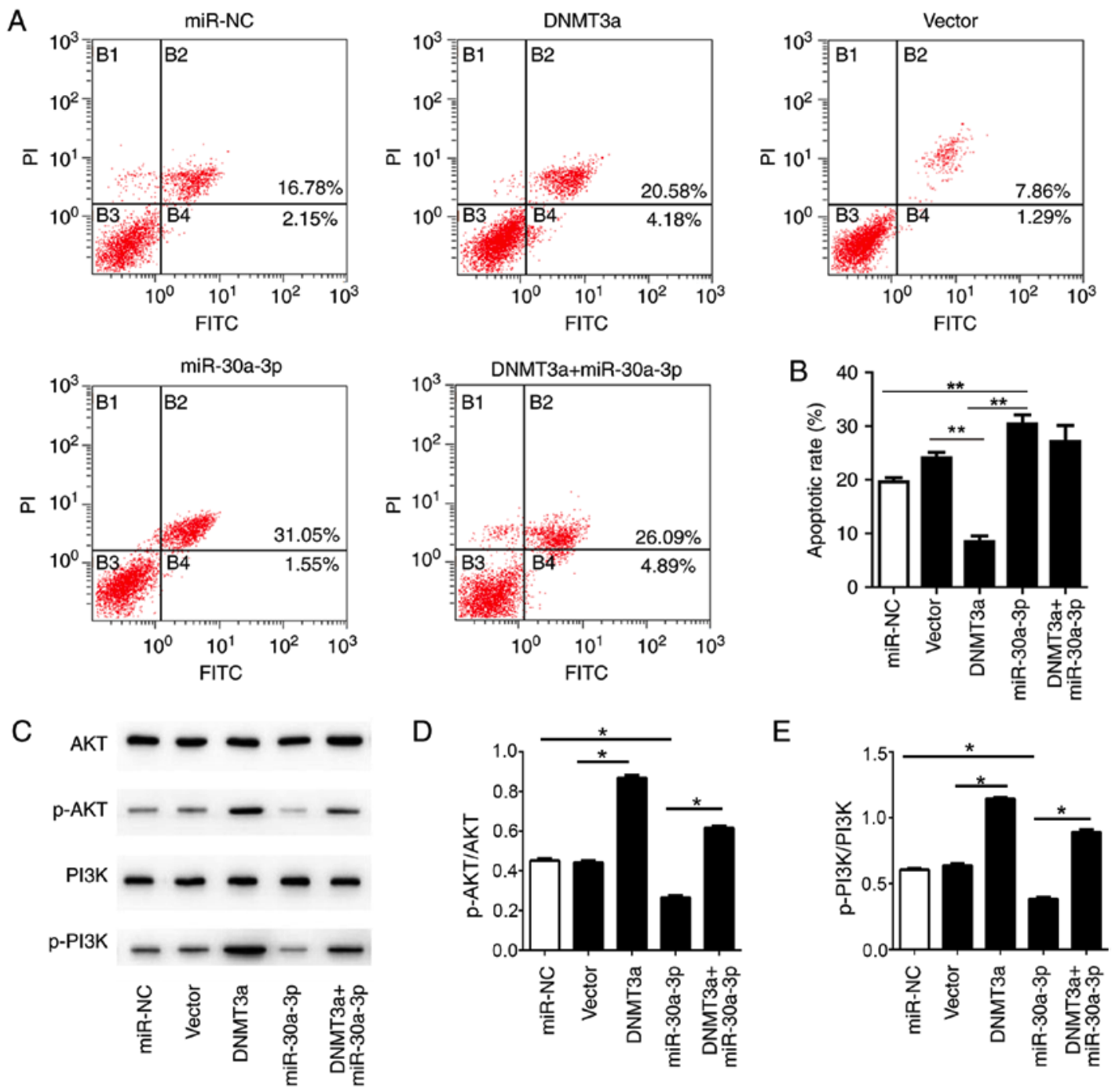

Figure 5. Overexpression of miR-30a-3p inhibits the anti-apoptotic effect of DNMT3a in HepG2 cells. (A) Following transfection with miR-30a-3p mimic, pCMV6-AC-DNMT3a, co-transfection, miR-NC and empty vector, the cells were analyzed by flow cytometry to evaluate the apoptotic rate. (B) Statistical analysis of the apoptotic rate in the HepG2 cells. (C) The levels of AKT, p-AKT, PI3K and p-PI3K were analyzed by western blotting. Relative expression levels of (D) p-AKT/AKT, (E) p-PI3K/PI3K were analyzed statistically. The data are presented as the mean \pm standard deviation of three independent experiments. " $\mathrm{P}<0.05,{ }^{* *} \mathrm{P}<0.01$. NC, negative control; p, phosphorylated; DNMT3a, DNA methyltransferase 3a; miR, microRNA; PI, propidium iodide; PI3K, phosphoinositide 3-kinase.

pCMV6-AC-DNMT3a (Fig. 5C-E). The data suggest that miR-30a-3p could inhibit the levels of $\mathrm{p}-\mathrm{AKT}$ and $\mathrm{p}-\mathrm{PI} 3 \mathrm{~K}$, thereby promoting cell apoptosis. Meanwhile, miR-30a-3p was also observed to affect the function of DNMT3a. In summary, these data indicate that miR-30a-3p mediates cell apoptosis through the PI3K/AKT signaling pathway.

\section{Discussion}

The present study aimed to investigate the potential role of miR-30a-3p in liver cancer cells and to identify its detailed mechanism of action. Li et al (30) reported that miR-200b expression levels were significantly decreased in the HCC tissue samples and the HepG2 cell line. The results of the present study demonstrated that miR-30a-3p expression was significantly reduced in HepG2 cells. Additionally, the DNMT3a expression level in HepG2 cells was significantly increased compared with that in L02 cells. The present study determined that the mechanism underlying the combined effect of miR-30a-3p and DNMT3a involved regulation of hepatoblastoma cell proliferation via the PI3K/AKT signaling pathway.

A number of studies have demonstrated that DNMT3a is a target gene of a variety of miRNAs, including miR-200b (30), miR-29a/b/c (7,31), miR-143 (32) and miRNA-101 (33). The present study indicated that DNMT3a is a direct target gene of miR-30a-3p by a luciferase reporter assay. Wang et al (34) demonstrated that miR-30a-3p regulates vimentin, E-cadherin and matrix metalloproteinase- 3 in HCC and acts as a tumor suppressor in vitro. Recently, Liu et al (35) demonstrated that miR-30a-3p inhibits COX-2 expression and regulates the nuclear translocation of $\beta$-catenin, as well as acting as a tumor suppressor in gastric cancer. The present study further investigated the role of miR-30a-3p and DNMT3a in HepG2 cells, and observed that the level of miR-30a-3p was decreased and the expression of DNMT3a was increased in HepG2 cells. 
These results are consistent with those of a previous study, which revealed that DNMT3a serves an essential role in multiple tumors $(24,36)$. Similarly, the present study demonstrated that the upregulation of miR-30a-3p inhibited DNMT3a expression and cell proliferation. Furthermore, upregulation of miR-30a-3p reduced the number of cells in the $S$ phase and promoted cell apoptosis. By contrast, DNMT3a demonstrated the adverse effect in HepG2 cells, which could be rescued by miR-30a-3p. In summary, the results of the present study demonstrated that miR-30a-3p and DNMT3a function as tumor suppressors in HepG2 cells through negative feedback.

Li et al (37) reported that overexpression of DNMTs may result in hyper- methylation/inactivation of p16 and indirectly regulate the progression of HCC. Depletion of DNMT3a in the HCC cell line SMMC-7721 inhibited cell proliferation and decreased colony formation by demethylation of the PTEN promoter (38). Additionally, DNMT3a was responsible for downregulating miR-105 which promotes gastric cancer cell proliferation (39). The present study further investigated the underlying mechanism of miR-30a-3p and DNMT3a in HepG2 cells, and observed that DNMT3a promoted the expression of cell cycle-associated proteins cyclin D1 and CDKs, inhibited p21 and p27, and regulated cell proliferation. Consistent with the trend in cell cycle alterations, miR-30a-3p reversed the expression patterns of cyclin D1, CDKs p21 and p27 induced by DNMT3a. In addition, p-PI3K and p-AKT were significantly increased following DNMT3a overexpression, whereas transfection with miR-30a-3p mimic markedly downregulated these proteins. Previous studies reported that the PI3K/AKT signaling pathway mediates $\mathrm{HB}$ cell proliferation and migration $(40,41)$. Recently, a number of studies have reported the roles of miRNAs and PI3K/AKT in regulating tumor cells. For example, miR-214 may activate the PI3K/Akt signaling pathway to regulate cell proliferation and apoptosis in ovarian cancer (42). In addition, miR-10a inhibits cell proliferation and migration, and promotes the apoptosis of breast cancer cells (23), and miR-20 inhibits cell proliferation and autophagy via the PI3K/AKT/mTOR signaling pathway (43).

In conclusion, the present study demonstrated that miR-30a-3p and DNMT3a interact to regulate hepatoblastoma cell proliferation via PI3K/AKT signaling. The results of the present study provide a novel insight into the mechanisms by which miR-30a-3p suppresses the proliferation of cancer cells. Therefore, miR-30a-3p is a promising target for the development and application of clinical treatments of liver cancer.

\section{Acknowledgements}

Not applicable.

\section{Funding}

This study was supported by the Hubei Natural Science Foundation of China (grant no. 2015CFC847).

\section{Availability of data and materials}

The datasets used and/or analyzed during the current study are available from the corresponding author on reasonable request.

\section{Authors' contributions}

QC and YG participated in the design of this work. QY was responsible for the cell culture. QC, QY, FT, PZ, SL, JL and HM performed the experiments and analyzed data. QC was involved in drafting the manuscript. HM revised the manuscript. All authors read and approved the final manuscript.

\section{Ethics approval and consent to participate}

Not applicable.

\section{Patient consent for publication}

Not applicable.

\section{Competing interests}

All the authors declare that they have no competing interests.

\section{References}

1. Liu Y, Wang Y, Sun X, Mei C, Wang L, Li Z and Zha X: miR-449a promotes liver cancer cell apoptosis by downregulation of Calpain 6 and POU2F1. Oncotarget 7: 13491-13501, 2016.

2. Azlin AH, Looi LM and Cheah PL: Tissue microarray immunohistochemical profiles of p53 and pRB in hepatocellular carcinoma and hepatoblastoma. Asian Pac J Cancer Prev 15: 3959-3963, 2014.

3. López-Terrada D, Cheung SW, Finegold MJ and Knowles BB: Hep G2 is a hepatoblastoma-derived cell line. Hum Pathol 40: 1512-1515, 2009.

4. Liu CY, Chen KF and Chen PJ: Treatment of liver cancer. Cold Spring Harb Perspect Med 5: a021535, 2015.

5. Yang G, Song Y, Zhou X, Deng Y, Liu T, Weng G, Yu D and Pan S: DNA methyltransferase 3, a target of microRNA-29c, contributes to neuronal proliferation by regulating the expression of brain-derived neurotrophic factor. Mol Med Rep 12: 1435-1442, 2015.

6. Cocozza S, Akhtar MM, Miele G and Monticelli A: CpG islands undermethylation in human genomic regions under selective pressure. PLoS One 6: e23156, 2011.

7. Cui H, Wang L, Gong P, Zhao C, Zhang S, Zhang K, Zhou R, Zhao Z and Fan H: Deregulation between miR-29b/c and DNMT3A is associated with epigenetic silencing of the CDH1 gene, affecting cell migration and invasion in gastric cancer. PLoS One 10: $\mathrm{e} 0123926,2015$.

8. Huang C, Liu H, Gong XL, Wu LY and Wen B: Effect of evodiamine and berberine on the interaction between DNMTs and target microRNAs during malignant transformation of the colon by TGF- $\beta 1$. Oncol Rep 37: 1637-1645, 2017.

9. Huang C, Liu H, Gong XL, Wu L and Wen B: Expression of DNA methyltransferases and target microRNAs in human tissue samples related to sporadic colorectal cancer. Oncol Rep 36: 2705-2714, 2016

10. Wang YC, Tang FY, Chen SY, Chen YM and Chiang EP: Glycine-N methyltransferase expression in HepG2 cells is involved in methyl group homeostasis by regulating transmethylation kinetics and DNA methylation. J Nutr 141: 777-782, 2011.

11. Kloosterman WP and Plasterk RH: The diverse functions of microRNAs in animal development and disease. Dev Cell 11: 441-450, 2006.

12. Zhou L, Liu F, Wang $X$ and Ouyang G: The roles of microRNAs in the regulation of tumor metastasis. Cell Biosci 5: 32, 2015.

13. Ambros V: The functions of animal microRNAs. Nature 431: 350-355, 2004.

14. Ding T, Xu JH, Sun MM, Zhu S and Gao J: Predicting microRNA biological functions based on genes discriminant analysis. Comput Biol Chem 71: 230-235, 2017.

15. Xu N, Zhang J, Shen C, Luo Y, Xia L, Xue F and Xia Q: Cisplatin-induced downregulation of miR-199a-5p increases drug resistance by activating autophagy in HCC cell. Biochem Biophys Res Commun 423: 826-831, 2012. 
16. Carthew RW and Sontheimer EJ: Origins and mechanisms of miRNAs and siRNAs. Cell 136: 642-655, 2009.

17. Jia XQ, Cheng HQ, Qian X, Bian CX, Shi ZM, Zhang JP Jiang BH and Feng ZQ: Lentivirus-mediated overexpression of microRNA-199a inhibits cell proliferation of human hepatocellular carcinoma. Cell Biochem Biophys 62: 237-244, 2012.

18. Wang Y, Chen FQ, Zhao M, Yang Z, Li J, Zhang S, Zhang W, Ye L and Zhang X: The long noncoding RNA HULC promotes liver cancer by increasing the expression of the HMGA2 oncogene via sequestration of the microRNA-186. J Biol Chem 292: 15395-15407, 2017.

19. Chen R, Liao JY, Huang J, Chen WL, Ma XJ and Luo XD: Downregulation of SRC kinase signaling inhibitor 1 (SRCIN1) expression by MicroRNA-32 promotes proliferation and epithelial-mesenchymal transition in human liver cancer cells. Oncol Res 26: 573-579, 2018.

20. Yin S, Fan Y, Zhang H, Zhao Z, Hao Y, Li J, Sun C, Yang J, Yang Z, Yang X, et al: Differential TGF $\beta$ pathway targeting by miR-122 in humans and mice affects liver cancer metastasis. Nat Commun 7: 11012, 2016.

21. Chen KJ, Hou Y, Wang K, Li J, Xia Y, Yang XY, Lv G, Xing XL and Shen F: Reexpression of Let-7g microRNA inhibits the proliferation and migration via K-Ras/HMGA2/snail axis in hepatocellular carcinoma. Biomed Res Int 2014: 742417, 2014.

22. Ma Y, She XG, Ming YZ and Wan QQ: miR-24 promotes the proliferation and invasion of HCC cells by targeting SOX7. Tumour Biol 35: 10731-10736, 2014.

23. Ke K and Lou T: MicroRNA-10a suppresses breast cancer progression via PI3K/Akt/mTOR pathway. Oncol Lett 14: 5994-6000, 2017.

24. Li Y, Nie Y, Tu S, Wang H, Zhou Y, Du Y, Cao J and Ye M: Epigenetically deregulated miR-200c is involved in a negative feedback loop with DNMT3a in gastric cancer cells. Oncol Rep 36: 2108-2116, 2016.

25. Ingegnere T, Mariotti FR, Pelosi A, Quintarelli C, De Angelis B, Tumino N, Besi F, Cantoni C, Locatelli F, Vacca P and Moretta L: Human CAR NK cells: A new non-viral method allowing high efficient transfection and strong tumor cell killing. Front Immunol 10: 957, 2019.

26. Song H, Li D, Wu T, Xie D, Hua K, Hu J, Deng X, Ji C, Deng Y and Fang L: MicroRNA-301b promotes cell proliferation and apoptosis resistance in triple-negative breast cancer by targeting CYLD. BMB Rep 51: 602-607, 2018.

27. Livak KJ and Schmittgen TD: Analysis of relative gene expression data using real-time quantitative PCR and the 2(-Delta Delta C(T)) method. Methods 25: 402-408, 2001.

28. Zhang ZM, Lu R, Wang PC, Yu Y, Chen D, Gao L, Liu S, Ji D, Rothbart SB, Wang Y, et al: Structural basis for DNMT3A-mediated de novo DNA methylation. Nature 554: 387-391, 2018.

29. Zhou Y, Gu P, Shi W, Li J, Hao Q, Cao X, Lu Q and Zeng Y: MicroRNA-29a induces insulin resistance by targeting PPARd in skeletal muscle cells. Int J Mol Med 37: 931-938, 2016.

30. Li XY, Feng XZ, Tang JZ, Dong K, Wang JF, Meng CC, Wang J, Mo YW and Sun ZW: MicroRNA-200b inhibits the proliferation of hepatocellular carcinoma by targeting DNA methyltransferase 3a. Mol Med Rep 13: 3929-3935, 2016.
31. Zhu Y, Zheng G, Wang H, Jia Y, Zhang Y, Tang Y, Li W, Fan Y, Zhang X, Liu Y and Liu S: Downregulated miR-29a/b/c during contact inhibition stage promote 3T3-L1 adipogenesis by targeting DNMT3A. PLoS One 12: e0170636, 2017.

32. Zhang Q, Feng Y, Liu P and Yang J: MiR-143 inhibits cell proliferation and invasion by targeting DNMT3A in gastric cancer. Tumour Biol 39: 1010428317711312, 2017.

33. Wang L, Yao J, Sun H, He K, Tong D, Song T and Huang C: MicroRNA-101 suppresses progression of lung cancer through the PTEN/AKT signaling pathway by targeting DNA methyltransferase 3A. Oncol Lett 13: 329-338, 2017.

34. Wang W, Lin H, Zhou L, Zhu Q, Gao S, Xie H, Liu Z, Xu Z, Wei J, Huang $X$ and Zheng S: MicroRNA-30a-3p inhibits tumor proliferation, invasiveness and metastasis and is downregulated in hepatocellular carcinoma. Eur J Surg Oncol 40: 1586-1594, 2014.

35. Liu X, Ji Q, Zhang C, Liu X, Liu Y, Liu N, Sui H, Zhou L, Wang S and Li Q: miR-30a acts as a tumor suppressor by double-targeting COX-2 and BCL9 in H. pylori gastric cancer models. Sci Rep 7: 7113, 2017.

36. Lu W, Lu T and Wei X: Downregulation of DNMT3a expression increases miR-182-induced apoptosis of ovarian cancer through caspase-3 and caspase-9-mediated apoptosis and DNA damage response. Oncol Rep 36: 3597-3604, 2016.

37. Li H, Yang F, Gao B, Yu Z, Liu X, Xie F and Zhang J: Hepatitis $B$ virus infection in hepatocellular carcinoma tissues upregulates expression of DNA methyltransferases. Int J Clin Exp Med 8: 4175-4185, 2015.

38. Zhao Z, Wu Q, Cheng J, Qiu X, Zhang J and Fan H: Depletion of DNMT3A suppressed cell proliferation and restored PTEN in hepatocellular carcinoma cell. J Biomed Biotechnol 2010: $737535,2010$.

39. Zhou GQ, Han F, Shi ZL, Yu L, Li XF, Yu C, Shen CL, Wan DW, Zhu XG, Li R and He SB: DNMT3A-mediated down-regulation of microRNA-105 promotes gastric cancer cell proliferation. Eur Rev Med Pharmacol Sci 21: 3377-3383, 2017.

40. Xia Z, Zhang N and Ding D: Proliferation and migration of hepatoblastoma cells are mediated by IRS-4 via PI3K/Akt pathways. Int J Clin Exp Med 7: 3763-3769, 2014.

41. Esmaeili MA, Farimani MM and Kiaei M: Anticancer effect of calycopterin via PI3K/Akt and MAPK signaling pathways, ROS-mediated pathway and mitochondrial dysfunction in hepatoblastoma cancer (HepG2) cells. Mol Cell Biochem 397: 17-31, 2014.

42. Liu J, Chen W, Zhang H, Liu T and Zhao L: miR-214 targets the PTEN-mediated PI3K/Akt signaling pathway and regulates cell proliferation and apoptosis in ovarian cancer. Oncol Lett 14: 5711-5718, 2017.

43. He W and Cheng Y: Inhibition of miR-20 promotes proliferation and autophagy in articular chondrocytes by PI3K/AKT/mTOR signaling pathway. Biomed Pharmacother 97: 607-615, 2018.

This work is licensed under a Creative Commons Attribution-NonCommercial-NoDerivatives 4.0 International (CC BY-NC-ND 4.0) License. 